\title{
Traccianti ambientali: il campo termico, questo sconosciuto
}

\author{
M. Dolores Fidelibus \\ DICATECh - Dipartimento di Ingegneria Civile, Ambientale, del Territorio, Edile e di Chimica - Politecnico di Bari \\ d.fidelibus@poliba.it
}

Nell'ambito dell'approccio di tracciamento naturale delineato in Fidelibus (2012), nell'ambito di quelli definiti quali traccianti ambientali sono inclusi i traccianti fisici. Tra questi, la temperatura ha un ruolo molto importante (Anderson, 2005), anche se sottostimato in Italia nella prassi di ricerca in campo idrogeologico. La temperatura è evidentemente il parametro principe nel campo della geotermia ad alta e media entalpia, ma la sua distribuzione spaziale e la sua evoluzione nel tempo hanno altrettanto valore a scale geotermiche inferiori. Proprio per questo vale la pena di dedicare qualche riflessione al tracciamento naturale nel campo della geotermia a bassa entalpia.

Infatti, le leggi che regolano all'attualità le concessioni di permessi di ricerca e di sfruttamento in campo geotermico, comunque prevalentemente dedicate alla norma dei comparti a media e alta entalpia, ma anche il complesso del dibattito apertosi recentemente in merito agli studi preliminari all'insediamento di sistemi geotermici a bassa entalpia, non menzionano o non sottolineano a sufficienza l'importanza della definizione delle caratteristiche del campo termico nei siti interessati dalle opere. Poiché queste riguardano il sottosuolo, gli studi geologici e idrogeologici classici sono indubbiamente fondamentali nella fase della loro progettazione preliminare: tali studi, tuttavia, andrebbero integrati dalla conoscenza approfondita della configurazione del campo termico, sia nell'insaturo sia nel saturo. Da questa conoscenza possono scaturire informazioni decisive per la scelta dei sistemi di sfruttamento più opportuni, così evitando la realizzazione di progetti che possono risultare al minimo inefficaci, se non addirittura dannosi.

Motivare adeguatamente queste affermazioni richiederebbe uno spazio adeguato all'ampiezza e alla complessità dei fenomeni di trasporto di calore nel sottosuolo. Si può provare comunque a dare una visione di tale complessità esaminando le due zone d'interesse dei sistemi geotermici a bassa entalpia, la zona vadosa e quella satura.

La distribuzione della temperatura nel sottosuolo in condizioni naturali (non antropizzate) riflette i flussi di energia termica provenienti da due fonti primarie, l'irraggiamento (ciclico) dall'alto, e il flusso di calore geotermico (quasi costante) dal basso. I regimi termici della zona vadosa nella sua parte più superficiale variano con la latitudine, l'altitudine, la topografia, le proprietà del suolo, il suo contenuto d'acqua, l'ombreggiatura, e la vegetazione: le fluttuazioni della temperatura in tale porzione del sottosuolo dipendono in massima parte dall'evoluzione diurna dell'irraggiamento e dagli scambi di calore con l'atmosfera. Le variazioni cicliche non sono più rinvenibili a una certa profondità, definita in gran parte dalla presenza/assenza, persistenza ed entità dei flussi d'infiltrazione e percolazione. Su scala annuale gli andamenti della temperatura nella zona vadosa più superficiale sono molto differenti da quelli che caratterizzano la zona vadosa più profonda: oltre la profondità sino alla quale si manifesta variabilità stagionale (dai 5 ai 30 metri dal p.c. in mezzi porosi insaturi), ci si aspetta una regione nella quale la temperatura cresce uniformemente per conduzione con la profondità in ragione del flusso di calore geotermico. In realtà nella zona vadosa più profonda vi è di solito una grande variazione spaziale di questo flusso di calore, dovuta alcune volte a variazioni spaziali del flusso geotermico, ma più spesso a variazioni spaziali del flusso di acque di percolazione.

Nella zona satura i flussi advettivi possono determinare variazioni molto importanti del flusso geotermico. Già dai primi anni del secolo scorso i geofisici maturarono un dubbio sulle misure di flusso di calore terrestre che si manifestava, anche a scala regionale, molto spesso inferiore a quello atteso dal puro flusso conduttivo. Venne in seguito ipotizzato che ciò che portava a una diminuzione sensibile del flusso di calore terrestre era il trasporto advettivo di calore operato dalle acque sotterranee.

Il ruolo del flusso advettivo delle acque sotterranee sul campo geotermico fu dimostrato da Domenico e Palciauskas nel 1973. Un campo idraulico che si sovrapponga a un campo termico porta a un'alterazione di quest'ultimo essenzialmente determinata dall'angolo tra le direzioni dei relativi gradienti. Il numero di Péclet che domina la soluzione analitica del problema definito da Domenico e Palciauskas (1973) dipende principalmente dalla conduttività idraulica del mezzo e dal carico idraulico medio della falda: per alti numeri di Péclet, l'advezione domina sulla conduzione e il campo geotermico ne è sensibilmente alterato. Le alterazioni più rilevanti si osservano quando le linee di flusso sono normali o quasi-normali alle isoterme (zone di ricarica o di drenaggio), mentre i disturbi meno importanti si manifestano nelle regioni dove le linee di flusso sono parallele alle isoterme. A causa di quest'alterazione del campo termico conduttivo da parte del campo idraulico, il gradiente geotermico aumenterà all'aumentare della profondità nelle aree d'alimentazione e diminuirà con la profondità nelle zone di drenaggio.

La significatività degli effetti dovuti all'advezione (definita forzata, cioè determinata da gradienti idraulici) sul regime termico, dipende da molti fattori idrogeologici, quali la configurazione tridimensionale della superficie freatica, la geometria del bacino, la grandezza e la distribuzione spaziale della permeabilità, l'anisotropia idraulica (si pensi ai sistemi 
fratturati e/o carsici) e la profondità del sistema di flusso; le perturbazioni del campo termico aumentano all'aumentare della permeabilità dell'acquifero o del suo spessore. Gli stessi fattori che controllano il sistema di flusso, la profondità della circolazione e la distribuzione delle aree di ricarica e drenaggio, governano anche il disturbo idrogeologico sul regime termico conduttivo. A tutto ciò devono aggiungersi altri fattori di disturbo, quali le influenze dovute a corpi idrici superficiali (fiumi, laghi, canali di bonifica, aree umide), alle acque d'intrusione marina negli acquiferi costieri, alle opere di prelievo e alle attività civili e industriali, e agli sversamenti di fluidi (legali e illegali).

Una vasta letteratura scientifica tratta del ruolo della temperatura negli studi idrologici e idrogeologici: Anderson (2005) propone una review di buona parte dei lavori fondamentali e fornisce un'utile guida per chi voglia approfondire l'argomento, anche se il lavoro non può tener conto della più recente $\mathrm{e}$ amplissima produzione, specialmente dedicata agli aspetti di modellazione del trasporto di calore, spesso proprio finalizzati a comprendere l'interazione tra sistemi di sfruttamento geotermico, attività antropiche e campi termici, advettivi e non.

Gli elementi di conoscenza in merito alle caratteristiche dei campi termici, pur se sinteticamente riportati, illustrano quantomeno la complessità dei fattori che le determinano: tale complessità indica che, sia nell'ambito della zona vadosa, sia in quello della zona satura, non é possibile definire a priori quale sia la distribuzione spaziale della temperatura e, soprattutto, date le numerose forzanti in gioco (cicliche e acicliche), quanto tale distribuzione sia stabile nel tempo. Basti pensare a quante diverse influenze può essere sottoposto il campo termico in un basso topografico costiero ad alta antropizzazione, con falda libera a bassa soggiacenza in clima arido o semi-arido, o quanto possa essere complesso uno scenario termico in area carsica.

Quasi come un mantra emerge anche nel contesto della geotermia a bassa entalpia la necessità della misura del parametro (temperatura) nelle 3D (ricostruzione del campo) e del monitoraggio periodico finalizzato ad accertare le fluttuazioni dello stesso. I profili termici lungo la verticale dell'acquifero rappresentano un mezzo d'indagine idoneo per verificare le condizioni termiche del sottosuolo e per ripetere senza costi elevati le misure nel tempo: essi sono di facile esecuzione, ma vanno interpretati con giudizio, specie in presenza di gradienti idraulici verticali. L'interpolazione prudente di dati da profili termici (Fabbri e Trevisan, 2005; Fidelibus et al., 2010) può fornire un quadro conoscitivo esauriente alle diverse scale d'interesse. Sorprende l'assenza d'informazioni di tale natura nelle carte geoenergetiche a scala regionale di cui qualche amministrazione si è già dotata.

Nel caso della bassa entalpia, quindi, le condizioni idrogeologiche e termiche dovrebbero nella pratica determinare le scelte di progetto per tutte le tipologie di opere. Elementi come il tipo di acquifero, la geometria del dominio, la soggiacenza, i carichi idraulici e i gradienti, la conduttività idraulica delle formazioni acquifere, la trasmissività, l'immagazzinamento, la qualità delle acque sotterranee e la loro vulnerabilità, ma soprattutto le caratteristiche del campo termico, pos- sono prospettare alcune importanti limitazioni progettuali. I progettisti dovrebbero essere consapevoli delle conseguenze e dei rischi di un'errata valutazione delle condizioni idrogeologiche e termiche: dovrebbero conoscere i concetti del trasporto di calore nel sottosuolo, i diversi fattori che influenzano il bilancio energetico, il ruolo delle acque di percolazione e sotterranee, e valutare attentamente i rischi ambientali connessi alle opere.

La più comune conseguenza di un'errata valutazione delle proprietà termiche del sottosuolo è l'incremento dei costi e il malfunzionamento dei sistemi geotermici: essi potrebbero rivelarsi non efficienti quanto previsto e potrebbero causare problemi dopo pochi anni di operatività. Le limitazioni da tenere in considerazione sono principalmente fisiche (clima, caratteristiche geologico/idrogeologiche, caratteristiche del campo termico), ma possono anche essere connesse ad altre condizioni dei siti, quali gli interessi concorrenti nell'uso del suolo e del sottosuolo. E' importante che tutte le limitazioni siano considerate preliminarmente nello stadio di fattibilità dei progetti: spesso accade che l'insediamento di opere civili anticipi la definizione chiara del tipo d'indagini preliminari che dovrebbero essere condotte e dei vincoli ai quali tali opere dovrebbero essere soggette, con la conseguenza che le opere proliferano, magari singolarmente accettabili, senza tuttavia riguardo ai possibili danni ambientali di più larga scala che la loro natura, concentrazione e mutua interferenza può comportare e di cui si ha contezza, purtroppo, solo successivamente.

\section{BIBLIOGRAFIA}

Anderson M.P. (2005). Heat as groundwater tracer. Groundwater 43:951-968, DOI: 10.1111/j.1745-6584.2005.00052.x

Domenico P.A., Palciauskas V.V. (1973). Theoretical analysis of forced convective heat transfer in regional ground-water flow. Geol Soc Am Bull 84:3803-3813

Fabbri P, Trevisani S. (2005). Spatial distribution of temperature in the low-temperature geothermal Euganean field (NE Italy): a simulated annealing approach. Geothermics, Volume 34 (5). 617-631, http:// dx.doi.org/10.1016/j.geothermics.2005.07.001.

Fidelibus M. (2012). Geochimica dell'idrosfera: come, quando e perché. Acque Sotterranee - Italian Journal of Groundwater (2012) ASr02008:061-062 - DOI:107343/AS-008-120018

Fidelibus M.D., Tulipano L., D’Amelio P. (2010). Convective thermal field reconstruction by ordinary kriging in karstic aquifers (Puglia, Italy): geostatistical analysis of anisotropy. In Navarro et al (Eds.), Advances in Research in Karst Media, Environmental Earth Sciences Series, 203-208, ISBN 978-642-12485-3, http://link.springer. com DOI:10.1007/978-3-642-12486-0_31, Springer-Verlag, Berlin Heidelberg. 